\title{
Biomarkers of Cardiovascular Disease in Patients With Ankylosing Spondylitis
}

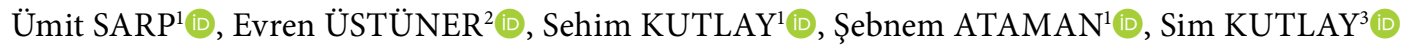 \\ ${ }^{1}$ Department of Physical Medicine and Rehabilitation, Ankara University School of Medicine, Ankara, Turkey \\ ${ }^{2}$ Department of Radiology, Ankara University School of Medicine, Ankara, Turkey \\ ${ }^{3}$ Department of Nephrology, Ankara University School of Medicine, Ankara, Turkey
}

\begin{abstract}
Objectives: This study aims to evaluate the inflammatory status and clinical and vascular alterations using tonometry and B-mode sonography in patients with ankylosing spondylitis (AS).

Patients and methods: The study included 71 AS patients (57 males, 14 females; mean age $40.1 \pm 10.8$ years; range, 29 to 51 years) without cardiovascular disease and 30 healthy controls ( 24 males, 6 females; mean age $41.2 \pm 9.3$ years; range, 32 to 50 years). We evaluated participants' clinical, biological and functional status along with laboratory data and measured both the arterial stiffness using carotid-femoral pulse wave velocity (PWV) measured by applanation tonometry and carotid intima-media thickness (CIMT) as a preclinical atherosclerosis marker.

Results: The mean disease duration of AS patients was $10.6 \pm 4.2$ years. CIMT $(p=0.03)$ and PWV $(p=0.04)$ data showed significant differences between AS patients and healthy controls. Multiregression analysis showed that PWV correlated with age $\left(r^{2}=0.42 ; p=0.03\right)$ and disease duration $\left(r^{2}=0.31 ; p=0.04\right)$, while CIMT correlated with disease duration $\left(r^{2}=0.37 ; p=0.03\right)$ and Bath Ankylosing Spondylitis Disease Activity Index $\left(r^{2}=0.3 ; p=0.04\right)$.

Conclusion: This study demonstrated an increase in early preclinical atherosclerosis in AS patients without cardiovascular (CV) disease compared to healthy controls. Therefore, screening AS patients with noninvasive methods for atherosclerosis and subclinical vasculopathy would allow us to take primary prevention measures. We found that the major determinant for increased CV risk was the disease duration, while there was no difference between different treatment modalities.

Keywords: Ankylosing spondylitis, arterial stiffness, cardiovascular disease, pulse wave velocity.
\end{abstract}

Patients with ankylosing spondylitis (AS), a chronic inflammatory disease affecting the spine and sacroiliac joints, ${ }^{1}$ have a higher risk for cardiovascular disease (CVD) compared to the general population. ${ }^{2}$ Though several traditional cardiovascular $(\mathrm{CV})$ risk factors like dyslipidemia, higher low-density lipoprotein cholesterol (LDL-C) to high-density lipoprotein cholesterol (HDL-C) ratio and hyperhomocysteinemia have been shown to be associated with $\mathrm{AS},{ }^{3,4}$ studies could not explain this increased risk solely with traditional $\mathrm{CV}$ risk factors. There is a sustained systemic inflammation in patients with AS with higher serum acute phase proteins such as $\mathrm{C}$-reactive protein (CRP), and systemic inflammation is linked with accelerated atherogenesis. ${ }^{5,6}$

Some mechanical arterial changes occur early in the development of atherosclerotic disease and these changes may be used to predict $\mathrm{CV}$ events in the future by the help of tests detecting such changes. The presence of subclinical vascular impairment can be evaluated by measuring aortic stiffness in the brachial artery simply and noninvasively using applanation tonometry. ${ }^{7}$

Received: October 20, 2019 Accepted: December 30, 2019 Published online: June 25, 2020

Correspondence: Sim Kutlay, MD. Ankara Üniversitesi Tıp Fakültesi Nefroloji Anabilim Dalı, 06590 Altındağ, Ankara, Türkiye. Tel: +90 505 - 6879185 e-mail: skutlay@hotmail.com

Sarp Ü, Üstüner E, Kutlay S, Ataman Ş, Kutlay S. Biomarkers of Cardiovascular Disease in Patients With Ankylosing Spondylitis. Arch Rheumatol 2020;35(3):435-439. 
By using a high resolution B-mode sonography, carotid intima-media thickness (CIMT) can also be measured. CIMT is one of the noninvasive markers of early atherosclerosis and it is the most popular way to assess the early structural changes in the arterial wall in the presence of atherosclerosis. ${ }^{8}$ In this study, we aimed to evaluate the inflammatory status and clinical and vascular alterations using tonometry and B-mode sonography in patients with AS.

\section{PATIENTS AND METHODS}

This study was conducted at Ankara University School of Medicine, Ibni Sina Hospital between March 2010 and January 2015. Seventy-one patients diagnosed as AS (57 males, 14 females; mean age $40.1 \pm 10.8$ years; range, 29 to 51 years) according to the modified New York diagnostic criteria and 30 healthy controls (24 males, 6 females; mean age $41.2 \pm 9.3$ years; range, 32 to 50 years) were included in the study. Of the patients, 30 (42\%) were on treatment with tumor necrosis factor (TNF) inhibitors for a median duration of 2.5 years (range, 1.5-5.3 years) and 41 (58\%) with nonsteroidal antiinflammatory drugs (NSAIDs). None of the patients received local or systemic corticosteroid medication within the last six months preceding the study. While $50(70.4 \%)$ of the 71 patients had only axial involvement, the remaining $(\mathrm{n}=21,29.6 \%)$ had peripheral arthritis. Patients with previous CVD or with a positive $\mathrm{CV}$ risk factor including hypertension, diabetes mellitus, hyperlipidemia, or smoking were excluded. Age- and sex-matched 30 healthy individuals with no CV risk factors, CVD or any rheumatic disease were included to form the control group. All participants in both groups were examined by electrocardiography and echocardiography to assess possible $\mathrm{CV}$ involvement and proved to be clinically relevant CVD free. None of the subjects in both groups received vasoactive drugs, statins, angiotensin converting enzyme inhibitors, calcium channel blockers, or anticoagulants, such as aspirin, clopidogrel, heparin or warfarin. The study protocol was approved by the Ankara University School of Medicine Ethics Committee. A written informed consent was obtained from each participant. The study was conducted in accordance with the principles of the Declaration of Helsinki.

Demographic, clinical, and functional characteristics of both AS and control groups (age, body mass index [BMI], disease duration, Bath Ankylosing Spondylitis Disease Activity Index [BASDAI] and functioning scores, used medications and smoking status) were obtained from the medical records. After overnight fasting, venous blood samples were obtained in the morning. Serum glucose, total cholesterol, HDL-C, LDL-C, triglyceride, renal and liver function tests, and a full blood count were measured using routine laboratory studies. Using a CRP reagent (Dialab, Vienna, Austria), serum was tested by nephelometry for the CRP level. Resting blood pressure was measured in sitting position with an automatic sphygmomanometer.

Arterial stiffness parameters were studied using a high-fidelity applanation tonometry (SphygmoCor device, AtCor Medical, Sydney, Australia). The patients were asked to be in fasting condition and to refrain from alcohol and coffee consumption for at least 10 hours. The measurements were performed in a quiet room with controlled temperature after 10 minutes in supine position. All measurements were performed twice by two trained technicians according to international recommendations. Another technician blinded to the clinical characteristics of the patients interpreted the results. ${ }^{9}$ Travel distance of the pulse wave was calculated by subtracting the distance from the suprasternal notch to the carotid pulse from the distance from the suprasternal notch to the femoral applanation tonometer. The carotidfemoral pulse wave velocity (PWV) measurement was an automatic calculation made by the device. The device calculated a corrected augmentation index (AIx) normalized for a heart rate of 75 beats per minute (AIx75) in order to obtain an AIx independent from individual pulse rate changes. ${ }^{10}$

The carotid arteries were imaged ultrasonically. A high-resolution ultrasound scanner (GE Medical Systems Norway AS, Oslo, Norway) with a 7-MHz linear array transducer was used to examine the right and left carotid arteries of the subjects in the supine position. The CIMT of the posterior wall was measured as the distance between the 
Table 1. Demographic and clinical characteristics of ankylosing spondylitis patients and control subjects

\begin{tabular}{|c|c|c|c|c|c|c|c|}
\hline & \multicolumn{3}{|c|}{ AS patients $(n=71)$} & \multicolumn{3}{|c|}{ Controls $(n=30)$} & \multirow[b]{2}{*}{$p$} \\
\hline & Mean \pm SD & Median & Min-Max & Mean \pm SD & Median & Min-Max & \\
\hline Age (year) & $40.1 \pm 10.8$ & & & $41.2 \pm 9.3$ & & & 0.61 \\
\hline Body mass index $\left(\mathrm{kg} / \mathrm{m}^{2}\right)$ & $25.8 \pm 2.4$ & & & $25.6 \pm 1.8$ & & & 0.9 \\
\hline Disease duration (year) & $10.6 \pm 4.2$ & & & & & & \\
\hline BASDAI & $4.0 \pm 2.8$ & & $2.2-6.7$ & & & & \\
\hline $\operatorname{ESR}(\mathrm{mm} / \mathrm{h})(\mathrm{CI})$ & $13.8 \pm 9-2$ & & & $4.8 \pm 4-7$ & & & 0.041 \\
\hline Triglyceride (mg/dL) & & 112.8 & $97-155$ & & 108.4 & $85-137$ & 0.56 \\
\hline LDL-C (mg/dL) & & 154.8 & $112-168$ & & 132.6 & $108-175$ & 0.6 \\
\hline C-reactive protein $(\mathrm{mg} / \mathrm{L})(\mathrm{CI})$ & $16.2 \pm 4.7$ & & $6.5-38.9$ & $1.6 \pm 0.3$ & & $1.0-2.5$ & 0.03 \\
\hline Glycemia (mg/dL) & $88.2 \pm 9.4$ & & $78-96$ & $91 \pm 6.9$ & & $83-95$ & 0.4 \\
\hline NSAIDS & & 41 & & & & & \\
\hline Biologic agents & & 30 & & & & & \\
\hline
\end{tabular}

borders of lumen-intima and media-adventitia in a region approximately $1 \mathrm{~cm}$ proximal to the carotid bifurcation. For each carotid artery, this measurement was repeated for four times at locations $1 \mathrm{~mm}$ apart. The mean value of the eight measurements was calculated (in $\mathrm{mm}$ ) and used for analysis.

\section{Statistical analysis}

All statistical analyses were conducted using the SPSS Statistics version 15.0 software (SPSS Inc., Chicago, IL, USA). For independent samples, non-parametric Mann-Whitney U test was used to compare data for patients and controls. To evaluate the association between the factors studied and arterial stiffness and CIMT in patients with AS, univariate (Pearson's or Spearman's correlation coefficient) and multivariate analyses were conducted with a $p$ value of $\leq 0.05$ considered as statistically significant.

\section{RESULTS}

The mean disease duration of the AS patients was $10.6 \pm 4.2$ years. The mean BASDAI score was $4.0 \pm 2.8$. Forty-one patients were receiving NSAID and 30 patients were receiving biological agents (etanercept, infliximab). Demographic characteristics, laboratory test results, and CV parameters for the patient and control groups are presented in Table 1.

There was no statistically significant difference in systolic and diastolic blood pressure, lipid profile, and glucose level between the patients

Table 2. Vascular parameters of ankylosing spondylitis patients and control subjects

\begin{tabular}{|c|c|c|c|c|c|c|c|}
\hline & \multicolumn{3}{|c|}{ AS patients $(n=71)$} & \multicolumn{3}{|c|}{ Controls $(n=30)$} & \multirow[b]{2}{*}{$p$} \\
\hline & Mean \pm SD & Median & Min-Max & Mean \pm SD & Median & Min-Max & \\
\hline Systolic blood pressure $(\mathrm{mmHg})$ & & 128 & $108-135$ & & 122 & $105-127$ & 0.5 \\
\hline Diastolic blood pressure $(\mathrm{mmHg})$ & & 82 & $72-84$ & & 79 & $72-83$ & 0.8 \\
\hline Pulse wave velocity $(\mathrm{m} / \mathrm{s})$ & & 8.82 & $7.0-10.0$ & & 6.6 & $6.4-7.1$ & $0.04^{*}$ \\
\hline Carotid intima media thickness $(\mathrm{mm})$ & $0.6 \pm 0.1$ & & & $0.6 \pm 0.2$ & & & $0.03^{*}$ \\
\hline Augmentation index (\%) & & 26.3 & 20.9-31.2 & & 18.9 & $15.8-21.6$ & $0.04^{*}$ \\
\hline Augmentation index 75 (\%) & & 25.2 & 18.9-30.2 & & 18.2 & $14.8-20.2$ & $0.03^{*}$ \\
\hline
\end{tabular}

AS: Ankylosing spondylitis; SD: Standard deviation; Min: Minimum; Max: Maximum 
and controls ( $p>0.05$ for all). ESR and CRP were significantly higher in the patients compared to the controls. In the AS group, mean symptom duration was $11.2 \pm 9.4$ years and mean diagnosis and treatment duration was $10.6 \pm 4.2$ years. BASDAI score was 4 or higher in $54 \%$ of the AS patients. The mean PWV, AIx, AIx75, and CIMT values were significantly higher in the patients compared to the controls (Table 2).

In the univariate analysis, PWV correlated with age $(r=0.4 ; p<0.05)$, systolic blood pressure $(\mathrm{r}=0.57 ; \mathrm{p}<0.05)$, BASDAI $(\mathrm{r}=0.4 ; \mathrm{p}<0.05)$, BMI $(r=0.34 ; \quad p<0.05)$, disease duration $(r=0.66$; $\mathrm{p}<0.01)$, and LDL $(\mathrm{r}=0.41 ; \mathrm{p}<0.05)$. AIx75 showed positive correlation with age $(\mathrm{r}=0.52$; $p<0.5)$ and disease duration $(r=0.57 ; p<0.01)$. CIMT correlated with age $(\mathrm{r}=0.44 ; \mathrm{p}<0.05)$, BASDAI $(r=0.36 ; p<0.05)$, disease duration $(\mathrm{r}=0.53 ; \mathrm{p}<0.05)$, CRP $(\mathrm{r}=0.33 ; \mathrm{p}<0.05)$, and HDL $(r=-0.26 ; p<0.05)$.

Multiregression analysis showed that PWV correlated with age $\left(r^{2}=0.42 ; 95 \%\right.$ confidence interval $[\mathrm{CI}]$ : $0.22-0.78 ; \mathrm{p}=0.03)$ and disease duration ( $r^{2}=0.31 ; 95 \%$ CI: 0.02-0.58; $\left.p=0.04\right)$, while CIMT correlated with disease duration $\left(r^{2}=0.37 ; 95 \%\right.$ CI: 0.10-0.34; $\left.p=0.03\right)$ and BASDAI $\left(r^{2}=0.3\right.$; 95\% CI: 0.18-0.38; $\left.\mathrm{p}=0.04\right)$.

\section{DISCUSSION}

We have shown an increase in arterial stiffness (expressed by PWV and AIx) and CIMT in patients with AS compared to age- and sex-matched healthy controls. Furthermore, PWV and CIMT showed a significant correlation with disease duration. PWV was also correlated with age while CIMT was correlated with BASDAI.

The detection of subclinical atherosclerosis in patients with AS has been a very attractive area of research since CVD is a major cause of death in these patients. We may adjust the treatment modality and reduce adverse outcomes by using tests used to detect subclinical atherosclerosis early in these patients. Unfortunately, there are conflicting reports regarding the association between AS and CIMT, and PWV.

Reported findings of the limited number of studies conducted to assess vascular function in patients with AS are controversial. There are studies, including those conducted by Sari et al., ${ }^{5}$ Malesci et al., ${ }^{3}$ and Choe et al., ${ }^{11}$ reporting no difference in CIMT between patients with AS and healthy controls. Unfortunately, the number of cases in these groups was under 30 . Controversially, Mathieu et al., ${ }^{12}$ Peters et al., ${ }^{1}$ and Bodnar et al. ${ }^{13}$ reported significantly higher CIMT values in patients with AS compared to controls. We reported an increase in CIMT correlated with PWV in 71 patients with AS. We found that the major factor affecting this increase was the duration of disease. There was no relationship with different treatment groups, CRP, and disease activity markers. This may be due to the changes in inflammatory status caused by the used medications.

Both genetic factors and chronic systemic inflammation act together in the development of atherosclerosis. Dysregulation of the innate immune pathway decreases soluble receptor for advanced glycation end products that could block the activation of the inflammatory pathway. The developing inflammation has adverse effects on blood vessels. ${ }^{14}$ Many proinflammatory factors are produced by macrophages, fibroblasts, dendritic cells, and endothelial cells. The increase in these proinflammatory factors (TNF-alpha, interleukin [IL]-6, IL-1) inhibits new collagen synthesis. Matrix metalloproteinases can degrade the extracellular matrix and this causes an imbalance between elastin and collagen. Processes that can increase CIMT also take place. The presence of chronic inflammation may increase the release of reactive oxygen species, which stimulate survival, migration, and proliferation of smooth cells in the arterial intima. ${ }^{15-17}$ On the other hand, disorders in lipid metabolism play an important role in the formation of atherosclerosis.

We attribute the strength of this study to the use of two noninvasive techniques to detect atherosclerosis and stiffness in the same AS population. We correlated the data we obtained with several clinical and laboratory measurements. Unfortunately, the relatively small number of patients and a possible selection bias of participants are the major limitations. Data on disease activity and use of medications between baseline and 10-year follow-up are also lacking. Since we did not assess baseline arterial stiffness and CIMT, we 
could not conclude on the progression of these two parameters.

In conclusion, this study demonstrated an increase in early preclinical atherosclerosis in AS patients without CVD compared to healthy controls. Therefore, screening AS patients with noninvasive methods for atherosclerosis and subclinical vasculopathy may be helpful. We found that the major determinant for increased $\mathrm{CV}$ risk was the disease duration, while there was no difference between different treatment modalities.

\section{Declaration of conflicting interests}

The authors declared no conflicts of interest with respect to the authorship and/or publication of this article.

\section{Funding}

The authors received no financial support for the research and/or authorship of this article.

\section{REFERENCES}

1. Peters MJ, van der Horst-Bruinsma IE, Dijkmans BA, Nurmohamed MT. Cardiovascular risk profile of patients with spondylarthropathies, particularly ankylosing spondylitis and psoriatic arthritis. Semin Arthritis Rheum 2004;34:585-92.

2. Szabo SM, Levy AR, Rao SR, Kirbach SE, Lacaille D, Cifaldi M, et al. Increased risk of cardiovascular and cerebrovascular diseases in individuals with ankylosing spondylitis: a population-based study. Arthritis Rheum 2011;63:3294-304.

3. Malesci D, Niglio A, Mennillo GA, Buono R, Valentini G, La Montagna G. High prevalence of metabolic syndrome in patients with ankylosing spondylitis. Clin Rheumatol 2007;26:710-4.

4. Başkan BM, Sivas F, Aktekin LA, Doğan YP, Ozoran K, Bodur H. Serum homocysteine level in patients with ankylosing spondylitis. Rheumatol Int 2009;29:1435-9.

5. Sari I, Okan T, Akar S, Cece H, Altay C, Secil $M$, et al. Impaired endothelial function in patients with ankylosing spondylitis. Rheumatology (Oxford) 2006;45:283-6.

6. van Eijk IC, Peters MJ, Serné EH, van der HorstBruinsma IE, Dijkmans BA, Smulders YM, et al. Microvascular function is impaired in ankylosing spondylitis and improves after tumour necrosis factor alpha blockade. Ann Rheum Dis 2009;68:362-6.

7. Avram C, Dragoi RG, Popoviciu H, Dragoi M, Avram A, Amaricai E. Association between arterial stiffness, disease activity and functional impairment in ankylosing spondylitis patients: a cross-sectional study. Clin Rheumatol 2016;35:2017-22.

8. Gonzalez-Gay MA, Gonzalez-Juanatey C, VazquezRodriguez TR, Martin J, Llorca J. Endothelial dysfunction, carotid intima-media thickness, and accelerated atherosclerosis in rheumatoid arthritis. Semin Arthritis Rheum 2008;38:67-70.

9. Nichols WW. Clinical measurement of arterial stiffness obtained from noninvasive pressure waveforms. Am J Hypertens 2005;18:3S-10S.

10. Laurent S, Cockcroft J, Van Bortel L, Boutouyrie P, Giannattasio C, Hayoz D, et al. Expert consensus document on arterial stiffness: methodological issues and clinical applications. Eur Heart $\mathrm{J}$ 2006;27:2588-605.

11. Choe JY, Lee MY, Rheem I, Rhee MY, Park SH, Kim SK. No differences of carotid intima-media thickness between young patients with ankylosing spondylitis and healthy controls. Joint Bone Spine 2008;75:548-53.

12. Mathieu S, Joly H, Baron G, Tournadre A, Dubost JJ, Ristori JM, et al. Trend towards increased arterial stiffness or intima-media thickness in ankylosing spondylitis patients without clinically evident cardiovascular disease. Rheumatology (Oxford) 2008;47:1203-7.

13. Bodnár N, Kerekes G, Seres I, Paragh G, Kappelmayer J, Némethné ZG, et al. Assessment of subclinical vascular disease associated with ankylosing spondylitis. J Rheumatol 2011;38:723-9.

14. Bai R, Zhang Y, Liu W, Ma C, Chen X, Yang J, et al. The Relationship of Ankylosing Spondylitis and Subclinical Atherosclerosis: A Systemic Review and Meta-Analysis. Angiology 2019;70:492-500.

15. Schuett H, Oestreich R, Waetzig GH, Annema W, Luchtefeld M, Hillmer A, et al. Transsignaling of interleukin-6 crucially contributes to atherosclerosis in mice. Arterioscler Thromb Vasc Biol 2012;32:281-90.

16. Lin J, Kakkar $\mathrm{V}$, Lu X. Impact of matrix metalloproteinases on atherosclerosis. Curr Drug Targets 2014;15:442-53.

17. Hoshi T, Kitagawa K, Yamagami H, Furukado S, Hougaku H, Hori M. Relation between interleukin-6 level and subclinical intracranial large-artery atherosclerosis. Atherosclerosis 2008;197:326-32. 